PROCEEDINGS OF THE

AMERICAN MATHEMATICAL SOCIETY

Volume 129, Number 1, Pages 97-103

S 0002-9939(00)05489-7

Article electronically published on June 21, 2000

\title{
BETWEEN THE LINDELÖF PROPERTY AND COUNTABLE TIGHTNESS
}

\author{
R. FRANKIEWICZ, G. PLEBANEK, AND C. RYLL-NARDZEWSKI
}

(Communicated by Dale Alspach)

\begin{abstract}
We consider a class of compact spaces $K$ for which the space $P(K)$ of probability Radon measures on $K$ has countable tightness in the weak* topology. We show that that class contains those compact zero-dimensional spaces for which $C(K)$ is weakly Lindelöf, and, under MA $+\neg \mathrm{CH}$, all compact spaces $K$ with $C(K)$ having property (C) of Corson.
\end{abstract}

\section{INTRODUCTION}

For an arbitrary set $X$ we denote by $[X] \leq \omega$ the family of all at most countable subsets of $X$. Recall that a topological space $X$ is said to have countable tightness at point $x \in X$ if for every set $A \subseteq X$ with $x \in \bar{A}$ there is $I \in[A] \leq \omega$ such that $x \in \bar{I}$; in such a case we write $t(x, X)=\omega$. We say that the space $X$ has countable tightness and write $t(X)=\omega$ if $t(x, X)=\omega$ for every $x \in X$.

Let $X$ be a completely regular Hausdorff topological space, and let $C_{p}(X)$ be the space of continuous functions on $X$ endowed with the topology of pointwise convergence. According to a theorem due to Arhangel'skil and Pytkeev (see Theorem II.1.1 of [1] $), t\left(C_{p}(X)\right)=\omega$ if and only if $X^{n}$ is Lindelöf for every $n$. That result led to the following problem:

Assume that $X$ is Lindelöf; is it true that every compact subspace of $C_{p}(X)$ has countable tightness?

This interesting and apparently difficult question has been answered in positive by Arhangel'skiı [1], Theorem IV.11.14, assuming the Proper Forcing Axiom. It seems that no further solutions are known (cf. [3]). It is even not known if one can show in ZFC that the space $\beta \omega$ cannot be embedded into $C_{p}(X)$, with $X$ being a Lindelöf space (see Arhangel'skiı̌ [2], Problem 52; cf. Cascales, Manjabacas and Vera [4]).

One can also consider the following version of the problem above: Let $E$ be a Banach space which is Lindelöf in its weak topology; is it true that the unit ball in $E^{*}$ has countable tightness in its weak* topology? This is partially related to a problem posed by Corson, if the product of weak topologies on $E \times E$ is Lindelöf provided $E$ is weakly Lindelöf (cf. [13]). In such a setting, it is perhaps more natural to consider property (C) of Corson rather than weak Lindelöfness itself (see [17]).

Received by the editors July 22, 1998 and, in revised form, March 8, 1999

2000 Mathematics Subject Classification. Primary 46E15, 46E27, 54C35.

This research was partially supported by KBN grant 2P03A 01813. 
The present paper deals with an even more concrete question: for which compact spaces $K$, does the space $P(K)$ (of probability Radon measures on $K$ ) have countable tightness in its weak* topology? We show below that the tightness of $P(K)$ is countable whenever $C(K)$ is weakly Lindelöf, under an additional assumption that $K$ is zero dimensional. Moreover, using a recent result of Fremlin [9], we show that assuming Martin's axiom and the negation of $\mathrm{CH}, P(K)$ has countable tightness provided $C(K)$ has property $(\mathrm{C})$ of Corson. Our theorems (partially) extend a result due to Pol [18.

\section{Some properties of $C(K)$ And $P(K)$}

In the sequel, $K$ always stands for a compact Hausdorff topological space. For a given space $K$, we denote by $C(K)$ the Banach space of continuous functions on $K$. As usual, the conjugate space $C(K)^{*}$ is identified with $M(K)$, the space of signed Radon measures on $K$ of finite variation. We denote by $P(K)$ the set of probability Radon measures on $K$. We always consider $P(K)$ with its weak* topology.

Note that the tightness of $P(K)$ is the same as the tightness of the unit ball $M_{1}(K)$ in $M(K)$. It is clear that $t(P(K)) \leq t\left(M_{1}(K)\right)$. On the other hand, denoting $\{(a, b):|a|+|b| \leq 1\}$ by $D$, we have a continuous surjection $\theta: P(K) \times$ $P(K) \times D \longrightarrow M_{1}(K)$ given by $\theta(\mu, \nu, a, b)=a \mu-b \nu$, so, using 3.12 .8 (f),(a) of [7] we get $t\left(M_{1}(K)\right) \leq t(P(K))$.

Let $E$ be a Banach space equipped with its weak topology. Corson [5] introduced the following convex analogue of the Lindelöf property: Say that $E$ has property (C) if for every family $\mathcal{C}$ of closed convex subsets of $E$ we have $\bigcap \mathcal{C} \neq \emptyset$ provided every countable subfamily of $\mathcal{C}$ has nonempty intersection.

Clearly, $E$ has property (C) whenever $E$ is weakly Lindelöf (recall that closed= weakly closed for convex sets). The converse does not hold: Pol 17 showed that if $K$ is the "two arrows space", then the space $C(K)$ has property (C) but is not weakly Lindelöf.

Pol 17] showed that $E$ has property (C) if and only if the weak* topology on $E^{*}$ satisfies a certain condition of "convex countable tightness". If $E=C(K)$, that result reads as follows (cf. Pol [18], Lemma 3.2).

Theorem 2.1 (R. Pol). For a compact space $K$ the following are equivalent:

(i) the space $C(K)$ has property $(C)$;

(ii) whenever $\mu \in \overline{\mathfrak{M}}$, where $\mathfrak{M} \subseteq P(K)$, then there is $\mathfrak{N} \in[\mathfrak{M}] \leq \omega$ such that $\mu \in \overline{\operatorname{conv} \mathfrak{N}}$.

It is not clear whether condition (ii) from the theorem above is equivalent to saying that $P(K)$ has countable tightness (for arbitrary compact space $K$ ). This problem was investigated by $\mathrm{Pol}$ [18, who in particular showed the following.

Theorem 2.2 (R. Pol). Assume that $K$ is a compact space such that every $\mu \in$ $P(K)$ is countably determined. Then the space $C(K)$ has property $(C)$ if and only if $t(P(K))=\omega$.

A measure $\mu \in P(K)$ is called countably determined if there is a countable family $\mathcal{F}$ of compact subsets of $K$ such that $\mu(B)=\sup \{\mu(F): F \subseteq B, F \in \mathcal{F}\}$ for every open set $B$. For arbitrary compact space $K$ the following implications hold (cf. Pol [17, 18]):

$$
t(P(K))=\omega \Longrightarrow C(K) \text { has property }(\mathrm{C}) \Longrightarrow t(K)=\omega
$$


Indeed, the first implication follows from Theorem 2.1 while the second may be checked in a straightforward way (given $A \subseteq K, x \in \bar{A}$, consider sets $\{g \in C(K)$ : $\left.g_{\mid B}=0, g(x)=1\right\}$, where $\left.B \in[A] \leq \omega\right)$. Moreover, if $C(K)$ has property (C) then every measure $\mu \in P(K)$ has a separable support. Indeed, otherwise there is a measure $\mu \in P(K)$ that vanishes on all separable closed subset of $K$, and considering the family $\left(C_{x}\right)_{x \in K}$, where

$$
C_{x}=\{g \in C(K): g(x)=0, \mu(g) \geq 1\},
$$

we see that $C(K)$ fails to have property $(\mathrm{C})$.

Haydon [10] and Kunen [11] presented closely related results that are relevant here. They, in particular, showed that assuming the continuum hypothesis there exist a first-countable compact space $K$ and a measure $\mu \in P(K)$ such that $L_{1}(\mu)$ is nonseparable and $\mu(M)=0$ whenever $M \subseteq K$ is separable. In fact the resulting space $K$ is Corson compact; see also [12] and [16] for similar constructions carried out under weaker axioms. As noticed in [17], for such a space $K$ we have $t(K)=\omega$ while $C(K)$ fails to have property $(\mathrm{C})$, and thus $P(K)$ has uncountable tightness.

It seems unknown whether the implication

$$
t(K)=\omega \Longrightarrow C(K) \text { has property }(\mathrm{C})
$$

is relatively consistent. We shall show in the next section that, assuming Martin's axiom and the negation of the continuum hypothesis, if $C(K)$ has property $(\mathrm{C})$, then $t(P(K))=\omega$.

We enclose here the result not directly related to our main subject. Note that property $(\mathrm{C})$ of a Banach space $E$ may be weakened to saying that $E$ is realcompact in its weak topology (that is, every maximal filter in the family of weak zero subsets of $E$, closed under countable intersection, is fixed); see Corson [5] and Edgar [6] for further information. The argument below closely follows the proof of Theorem 3.1 from [14; see also Plebanek [15].

Theorem 2.3. If $K$ is a compact space of countable tightness, then the Banach space $C(K)$ is realcompact in its weak topology.

Proof. We shall check that if $z \in C(K)^{* *}$ is a functional which is weak $k^{*}$ continuous on every weak ${ }^{*}$ separable subspace of $C(K)^{*}$, then $z \in C(K)$. According to a result due to Corson [5], this characterizes realcompactness of the weak topology on $C(K)$.

Denote by $\delta_{x} \in P(K)$ the Dirac measure at $x \in K$. Define a function $\varphi$ on $K$ by the formula $\varphi(x)=z\left(\delta_{x}\right)$. Note that $\varphi$ is continuous on every separable subspace of $M$ of $K$, since $z$ is weak $k^{*}$ continuous on the subspace spanned by $\left(\delta_{x}\right)_{x \in M}$. Since $t(K)=\omega$, it follows that $\varphi \in C(K)$. Now it suffices to check that $z(\mu)=\mu(\varphi)$ for every $\mu \in P(K)$; in other words we are to prove that $w=z-\varphi=0$.

Suppose the contrary; we may then assume that there is $\mu \in P(K)$ with $w(\mu)>$ 0 . Given a Borel set $B \subseteq K$, denote by $\mu_{B}$ the restriction of a measure $\mu$ to $B$. Using the Radon-Nikodym theorem to the signed $\mu$-continuous measure $B \rightarrow w\left(\mu_{B}\right)$ we get a $\mu$-measurable function $g$ on $K$ with $w\left(\mu_{B}\right)=\int_{B} g \mathrm{~d} \mu$ for every Borel set $B$. Take $c>0$ and a closed set $M \subseteq\{g \geq c\}$ so that $\mu(M)>0$. Now the measure $\nu=\frac{1}{\mu(M)} \cdot \mu_{M}$ is such that $w\left(\nu_{B}\right) \geq c \nu(B)$ for all Borel sets $B$. We may assume that $M$ is self-supporting, that is $\mu(M \cap V)>0$ whenever $V \subseteq K$ is an open set with $V \cap M \neq \emptyset$. 
By a result due to Shapirovskiu [19], there is a point $x \in M$ which has countable $\pi$-character in $M$. That means that there is a sequence $\left(U_{n}\right)_{n \in \omega}$ of open subsets of $M$ such that for every neighbourhood of $x$ contains $U_{n}$ for some $n$. Letting $\nu_{n}=\frac{1}{\nu\left(U_{n}\right)} \nu_{U_{n}}$ and $\mathfrak{N}=\left(\nu_{n}\right)_{n \in \omega}$, one can easily check that $\delta_{x} \in \overline{\mathfrak{N}}$. Now we have $w\left(\nu_{n}\right) \geq c, w\left(\delta_{x}\right)=0$, and this is a contradiction, since the functional $w$ is weak $k^{*}$ continuous on $\overline{\mathfrak{N}}$.

\section{On Countable tightness of $P(K)$}

We shall see in this section that countable tightness of $P(K)$ is somewhat related to separability of measures from $P(K)$. We say that a measure $\mu \in P(K)$ is separable if $L_{1}(\mu)$ is separable as a Banach space. Clearly, every countably determined measure is separable.

For the sake of the proof of our first lemma we recall the following property of tightness: If $K$ is a compact space and $Z \subseteq K$ is a closed $\mathcal{G}_{\delta}$ set, then $t(x, Z)=$ $t(x, K)$ for every $x \in Z$ (see [7], 3.12.8(c)).

Lemma 3.1. Assume that $K$ is a compact space and $\mu \in P(K)$ is separable. If $t(\mu, P(K))>\omega$, then the Banach space $C(K)$ does not have property $(C)$.

Proof. We find and fix a countable family $\mathcal{F} \subseteq C(K)$ which is dense in $L_{1}(\mu)$. Applying the above remark to $\mu$ and the zero set

$$
\mathfrak{Z}=\bigcap_{f \in \mathcal{F}}\{\nu \in P(K): \nu(f)=\mu(f)\},
$$

we have $t(\mu, \mathfrak{Z})>\omega$. Thus we can find an uncountable family $\mathfrak{M} \subseteq \mathfrak{Z}$ such that $\mu \in \overline{\mathfrak{M}}$ while $\mu \notin \overline{\mathfrak{N}}$ whenever $\mathfrak{N} \in[\mathfrak{M}] \leq \omega$.

Given functions $g_{1}, \ldots, g_{k} \in C(K)$, and $\varepsilon>0$, we let

$$
V\left(g_{1}, \ldots, g_{k}, \varepsilon\right)=\left\{\nu \in P(K):(\forall i \leq k)\left|\nu\left(g_{i}\right)-\mu\left(g_{i}\right)\right|<\varepsilon\right\},
$$

which is a wea $k^{*}$-open neighbourhood of $\mu \in P(K)$.

Note that we can find a positive $\varepsilon$ such that for every $\mathfrak{N} \in[\mathfrak{M}] \leq \omega$ there are a number $n$ and functions $g_{1}, \ldots, g_{n} \in C(K)$ such that

$$
\mathfrak{N} \cap V\left(g_{1}, \ldots, g_{n}, 3 \varepsilon\right)=\emptyset .
$$

This follows from the fact that the closure of no countable family in $\mathfrak{M}$ contains $\mu$.

For any measure $\nu \in P(K)$ the set

$$
Z(\nu)=\{g \in C(K): \nu(g) \geq 2 \varepsilon, \mu(g) \leq \varepsilon\}
$$

is convex and closed in $C(K)$. Since $\mu \in \overline{\mathfrak{M}}$, we have $\bigcap_{\nu \in \mathfrak{M}} Z(\nu)=\emptyset$. We shall check that $C(K)$ does not have property (C) by showing that the set $\bigcap_{\nu \in \mathfrak{N}} Z(\nu)$ is nonempty whenever $\mathfrak{N} \in[\mathfrak{M}] \leq \omega$.

For a fixed countable $\mathfrak{N} \subseteq \mathfrak{M}$, we find $g_{1}, \ldots, g_{n} \in C(K)$ satisfying $(*)$. Letting $\delta=\varepsilon / n$, we take functions $f_{i} \in \mathcal{F}$ such that $\int\left|f_{i}-g_{i}\right| d \mu<\delta$ for $i=1,2, \ldots, n$. Now consider a function $h=\sum_{i=1}^{n}\left|f_{i}-g_{i}\right|$.

We have $\mu(h) \leq \delta n=\varepsilon$. On the other hand, for any $\nu \in \mathfrak{N}$ there is $j \leq n$ such that $\left|\nu\left(g_{j}\right)-\mu\left(g_{j}\right)\right| \geq 3 \varepsilon$, and hence

$$
\begin{gathered}
\nu(h) \geq\left|\nu\left(g_{j}\right)-\nu\left(f_{j}\right)\right| \geq\left|\nu\left(g_{j}\right)-\mu\left(g_{j}\right)\right|-\left|\nu\left(f_{j}\right)-\mu\left(g_{j}\right)\right| \\
\geq 3 \varepsilon-\left|\mu\left(f_{j}\right)-\mu\left(g_{j}\right)\right| \geq 2 \varepsilon .
\end{gathered}
$$

We have shown that $h \in \bigcap_{\nu \in \mathfrak{N}} Z(\nu)$, and this finishes the proof. 
The above lemma and Theorem 2.1 yield the following extension of Theorem 2.2.

Theorem 3.2. Assume that $K$ is a compact space such that every $\mu \in P(K)$ is separable. Then the space $C(K)$ has property $(C)$ if and only if $t(P(K))=\omega$.

As we mentioned before under $\mathrm{CH}$ there are first-countable compact spaces carrying nonseparable Radon measures. The situation is quite different under Martin's axiom (MA) and $\neg \mathrm{CH}$. The following result due to Fremlin [9] has solved so-called Haydon's problem (cf. [12], [16]).

Theorem 3.3 (D. Fremlin). Assume $M A$ and $\neg C H$. The following are equivalent for a compact space $K$ :

(i) there is a nonseparable measure $\mu \in P(K)$;

(ii) there is a continuous surjection from $K$ onto $[0,1]^{\omega_{1}}$.

In particular, if $t(K)=\omega$, then every measure $\mu \in P(K)$ is separable.

Theorem 3.4. Under $M A$ and $\neg C H$, the following two conditions are equivalent for every compact space $K$ :

(i) the space $P(K)$ has countable tightness;

(ii) the Banach space $C(K)$ has property $(C)$.

Proof. If $C(K)$ has property $(\mathrm{C})$, then $K$ has countable tightness (see the previous section). Hence every Radon measure on $K$ is separable by Theorem 3.3, and $t(P(K))=\omega$ by Theorem 3.2 .

Lemma 3.5. Let $K$ be a compact zero-dimensional space. If $C(K)$ is Lindelöf in its weak topology, then every measure $\mu \in P(K)$ is separable.

Proof. Suppose that there is a nonseparable Radon measure on $K$. We can then find a measure $\mu \in P(K)$ which is homogenous of uncountable Maharam type. By the Maharam theorem (see 8]), the measure algebra of $\mu$ contains a subalgebra isomorphic to the measure algebra of the usual product measure on $2^{\omega_{1}}$. It follows that one can find a family $\left(B_{\alpha}\right)_{\alpha<\omega_{1}}$ of Borel subsets of $K$ with $\mu\left(B_{\alpha}\right)=1 / 2$, which are independent with respect to $\mu$.

We fix a positive constant $\varepsilon \leq 1 / 16$. Next we find for every $\alpha<\omega_{1}$ a closed and open set $V_{\alpha} \subseteq K$, such that $\mu\left(B_{\alpha} \triangle V_{\alpha}\right)<\varepsilon$. Since

$$
V_{\alpha} \cap V_{\beta}^{c} \supseteq B_{\alpha} \cap B_{\beta}^{c} \backslash\left[\left(B_{\alpha} \triangle V_{\alpha}\right) \cup\left(B_{\beta}^{c} \triangle V_{\beta}^{c}\right)\right],
$$

we have $\mu\left(V_{\alpha} \cap V_{\beta}^{c}\right) \geq 1 / 4-2 \varepsilon$ whenever $\alpha \neq \beta$.

We let $\mathcal{F}$ be the weak closure in $C(K)$ of the family of continuous functions $\left(\chi_{V_{\alpha}}\right)_{\alpha<\omega_{1}}$. Note that every $f \in \mathcal{F}$ may be written as $f=\chi_{F}$, where $F \subseteq K$ is closed and open (and $|\mu(F)-1 / 2| \leq \varepsilon$ ). We define

$$
U(F)=\left\{g \in C(K): \int_{F^{c}} g \mathrm{~d} \mu<\varepsilon\right\},
$$

for every $\chi_{F} \in \mathcal{F}$. Obviously, $U(F)$ is a weak open neighbourhood of $\chi_{F}$. Thus $(U(F))_{\chi_{F} \in \mathcal{F}}$ is an open cover of $\mathcal{F}$; we shall prove that $\mathcal{F}$ (and hence $C(K)$ ) is not Lindelöf, by checking that no countable family of sets of the form $U(F)$ covers $\mathcal{F}$.

Consider a sequence $\left(F_{k}\right)_{k \in \omega}$ of sets, the characteristic functions of which lie in $\mathcal{F}$. For every $k$ there is $\alpha_{k}<\omega_{1}$ such that $\mu\left(F_{k} \backslash V_{\alpha_{k}}\right)<\varepsilon$. Indeed, the set

$$
\left\{g \in C(K): \int_{F_{k}} g \mathrm{~d} \mu>\mu\left(F_{k}\right)-\varepsilon\right\}
$$


is an open neighbourhood of $\chi_{F_{k}}$ so for some $\alpha_{k}<\omega_{1}$ we have

$$
\int_{F_{k}} \chi_{V_{\alpha_{k}}} \mathrm{~d} \mu>\mu\left(F_{k}\right)-\varepsilon
$$

which gives $\mu\left(F_{k} \cap V_{\alpha_{k}}\right)>\mu\left(F_{k}\right)-\varepsilon$, and $\mu\left(F_{k} \backslash V_{\alpha_{k}}\right)<\varepsilon$.

Now take any $\eta \in \omega_{1} \backslash\left\{\alpha_{k}: k \in \omega\right\}$. We have

$$
\mu\left(V_{\eta} \backslash F_{k}\right) \geq \mu\left(V_{\eta} \backslash V_{\alpha_{k}}\right)-\mu\left(F_{k} \backslash V_{\alpha_{k}}\right) \geq 1 / 4-2 \varepsilon-\varepsilon \geq \varepsilon .
$$

Hence $\int_{F_{k}^{c}} \chi_{V_{\eta}} \mathrm{d} \mu \geq \varepsilon$ for every $k$, which means that $\chi_{V_{\eta}} \notin \bigcup_{k \in \omega} U\left(F_{k}\right)$, and the proof is complete.

Lemma 3.1 and Lemma 3.5 give immediately the following.

Theorem 3.6. Let $K$ be a compact zero-dimensional space. If the Banach space $C(K)$ is Lindelöf in its weak topology, then $t(P(K))=\omega$.

We do not know if the above theorem holds true for an arbitrary compact space $K$ (not necessarily zero dimensional).

\section{REFERENCES}

[1] A.V. Arhangel'skiǔ, Topological Function Spaces (in Russian), Izd. Moskovskovo Univ. (1989); also Topological Function Spaces, Kluwer Academic Publishers (1992). MR 92i:54022

[2] A.V. Arhangel'skiǔ, $C_{p}$-theory, in: Recent progres in general topology, M. Husek, J. van Mill (edts), North Holland (1992). CMP 93:15

[3] A.V. Arhangel'skiı̆, Szeptycki, Tightness in compact subspaces of $C_{p}$ spaces, Houston J. Math. 23 (1997), 87-93.

[4] B. Cascales, G. Manjabacas, G. Vera, Fragmentability and compactness in $C(K)-$ spaces, Studia Math. 131 (1998), 73-87. MR 99d:46038

[5] H.H. Corson, The weak topology of a Banach space, Trans. Amer. Math. Soc. 101 (1961), 1-15. MR 24:A2220

[6] G.A. Edgar, Measurability in a Banach space II, Indiana Univ. Math. J. 28 (1979), 559-579. MR 81d:28016

[7] R. Engelking, General Topology, PWN, Warsaw (1977). MR 58:18316b

[8] D.H. Fremlin, Measure algebras, in: Handbook of Boolean algebras, J.D. Monk (ed.), NorthHoland 1989, Vol. III, Chap. 22. CMP 21:10

[9] D.H. Fremlin, On compact spaces carrying Radon measures of uncountable Maharam type, Fund. Math. 154 (1997), 295-304. MR 99d:28019

[10] R. Haydon, On dual $L^{1}$-spaces and injective bidual Banach spaces, Israel J. Math. 31 (1978) 142-152. MR 80e:46013

[11] K. Kunen, A compact L-space under CH, Topology Appl. 12 (1981), 283-287. MR 82h:54065

[12] K. Kunen, J. van Mill, Measures on Corson compact spaces, Fund. Math. 147 (1995), 61-72. MR 96c:54040

[13] J. Orihuela, On weakly Lindelöf Banach spaces, in: Progress in Functional Analysis, K.D. Bierstedt et. al. (edts.), Elsevier 1992. [MR 93e:46021

[14] G. Plebanek, On some properties of Banach spaces of continuous functions, Seminaire d'Initiation a l'Analyse (G. Choquet et al.), 31 (1991/92), Universite Paris VI. MR 95d:46018

[15] G. Plebanek, On Mazur Property and realcompactness in $C(K)$, in Topology, Measure and Fractals, C. Bandt et al. (eds.), Math. Research vol. 66, Akademie Verlag (1992). MR 94m:46046

[16] G. Plebanek, Nonseparable Radon measures and small compact spaces, Fund. Math. 153 (1997), 25-40. MR 98m:28025

[17] R. Pol, On a question of H.H. Corson and some related problems, Fund. Math. 109 (1980), 143-154. MR 82a:46022 
[18] R. Pol, Note on the spaces of regular probability measures whose topology is determined by countable subsets, Pacific J. Math. 100 (1982), 185-201. MR 83g:54024

[19] B.E Shapirovskiı̌, On the $\pi$-character and $\pi$-weight in compact Hausdorff spaces, Dokl. Akad. Nauk SSSR 223 (1975), 161-164 (in Russian); also: Soviet Math. Dokl. 16 (1975), 999-1033. MR 53:14380

Institute of Mathematics, Polish Academy of Sciences, Ul. Kopernika 18, 51-617 Wroceaw, Poland

E-mail address: rf@impan.gov.pl

Institute of Mathematics, University of WrocŁaw, Pl. Grunwaldzki 2/4, 50-384 WroCŁaw, Poland

E-mail address: grzes@math.uni.wroc.pl

Institute of Mathematics, Wroceaw Technical University and Institute of Mathematics, Polish Academy of Sciences, ul. Kopernika 18, 51-617 WrocŁaw, Poland

E-mail address: crn@graf.im.pwr.wroc.pl 